\title{
SEMIGROUPS OF PARTIAL ISOMETRIES
}

\author{
BY LAWRENCE J. WALLEN
}

Communicated by Paul Halmos, February 24, 1969

Let $\left\{S_{t}\right\}$ be the (strongly continuous) semigroup of operators on $L_{2}(0,1)$ defined as follows: if $0 \leqq t<1$, then

$$
\begin{aligned}
S_{t} f(x)_{t}^{3} & =0 \quad \text { if } x \leqq t, \\
& =f(x-t) \quad \text { if } t<x \leqq 1 ;
\end{aligned}
$$

if $t \geqq 1$, then $S_{t}=0$. The operators $S_{t}$ are related to the classical Volterra operator $J\left(J f(x)=\int_{0}^{x} f(t) d t\right)$ by the equation $J=\int_{0}^{1} S_{t} d t$ or, what comes to the same thing, $i \lambda J(I-i \lambda J)^{-1}=\int_{0}^{1} e^{i \lambda t} S_{t} d t$. These formulas, together with the uniqueness of the Fourier transform, permit one to pass readily from considerations concerning $J$ to those about $\left\{S_{t}\right\}$ and vice versa. This correspondence was used by Dixmier in [1] where, however, he considers these operators on $L_{1}$.

Let us note three properties of $\left\{S_{t}\right\}$ :

(a) each $S_{t}$ is a partial isometry,

(b) $S_{1}=0$ and $S_{t} \neq 0$ if $0 \leqq t<1$,

(c) $\left\{S_{t}\right\}$ is irreducible.

It turns out that (a), (b), and (c) characterize $\left\{S_{t}\right\}$ up to unitary equivalence. This is a special case of the following result.

THEOREM. If $\left\{W_{t}\right\}$ is a strongly continuous semigroup of operators on a Hilbert space and if $\left\{W_{t}\right\}$ satisfies (a) and (b), then a necessary and sufficient condition that $\left\{W_{t}\right\}$ be unitarily equivalent to a direct sum of $n$ copies of $\left\{S_{t}\right\}$ (n may be infinite) is that the von Neumann algebra generated by $\left\{W_{t}\right\}$ be a factor.

The detailed proof will be published elsewhere, but a sketch may be of interest. The basic fact used (see [2]), is that a nilpotent partial isometry, all of whose powers are partial isometries has a sort of Jordan decomposition. Such an operator is, in fact, the direct sum of operators having a matrix representation:

$$
\left(\begin{array}{llll}
0 & 0 & \cdots & 0 \\
I & 0 & \cdots & 0 \\
0 & I & \cdots & 0 \\
\vdots & & & \vdots \\
0 & & & \vdots \\
0 & & & 0
\end{array}\right)
$$


A careful analysis of this representation applied to $W_{2^{-k}}$ permits us to construct projections $E_{\xi}, \xi \geqq 0$, in the center of the von Neumann algebra generated by $\left\{W_{t}\right\}$, with the property that $W_{t} \mid \operatorname{ran} E_{\xi}=0$ if $t \geqq \xi$. The assumption that the algebra is a factor enables us to conclude that for $0 \leqq t \leqq 1, \operatorname{ker}\left(W_{t}\right)=\operatorname{ran}\left(W_{1-t}\right)$. It then follows easily that the minimal isometric dilation $\left\{V_{t}\right\}$ has no unitary part and that $H=\operatorname{ran}^{\perp}\left(V_{1}\right)$. Finally, the theorem is established by appealing to Cooper's characterization of isometric flows (see [3]).

\section{REFERENCES}

1. J. Dixmier, Les operateurs permutable a l'operateurs integral, Portugal. Math. 8 (1949), 73-84.

2. P. R. Halmos and L. J. Wallen, Powers of parital isometries, J. Math. Mech. (to appear).

3. J. L. B. Cooper, One-parameter semi-groups of isometric operators in Hilbert space, Ann. of Math. 48 (1947), 827-842.

University of HawaII, Honolulu, HawaIr 96822 